\title{
Focus on How Plants Engage With Beneficial Microorganisms While at the Same Time Restricting Pathogens
}

\section{MPMI Focus Issue Overview}

Plants live in a world filled with microbes, and spend their lives engaged in the delicate dance of nurturing beneficial interactions while simultaneously reducing disease-causing interactions. The question How do plants engage with beneficial microorganisms while at the same time restricting pathogens? was recently selected in a crowd-sourced effort as the top, unanswered question in the field of molecular plant-microbe interactions. Elaborating on this question and setting the stage for this focus issue, the Top10 review by Thoms et al. examines the way multiple inputs are integrated to initiate programs of immunity or mutualistic symbiosis, and how this shapes the microbiome. This comprehensive review describes the current landscape of the field, focusing on the plant-microbe-soil continuum but providing ideas for extending these concepts to leaves, where much of the research on immunity has centered. The authors provide a new framework for considering this question in terms of metabolic gating, dual receptor recognition, and integration of environmental signals, laying a solid foundation for future research.

Plants engage with microbes at varying levels of intimacy, from occasional interactions with microbes inhabiting the soil to those epiphytically colonizing leaf or root surfaces to endophytes living within plant tissues or endosymbiotically within plant cells. Legumes form this most intimate interaction with bacteria collectively known as rhizobia, inducing the formation of symbiotic nodules on roots, which the bacteria infect and in which they fix nitrogen inside plant cells in exchange for carbon. This massive infection by a symbiont is accompanied by a suppression of the plant defense response. Two papers in this issue examine the delicate balance between inhibiting pathogens and allowing entry to beneficials. In field settings, legumes interact both with mutualists and with pathogens, which can cause substantial damage to legume crops. In their review, Costa and colleagues present a comprehensive overview of the varied responses of different legume species to simultaneous interactions with symbiotic rhizobia and parasitic root-knot nematodes, discussing research ranging from the molecular level to field applications. Benezech et al. focus on the complex response of Medicago truncatula to pathogenic Ralstonia solanacearum, examining the role of the Ralstonia type III secretion system (T3SS) in the inhibition of simultaneous infection by the rhizobial symbiont. The flip side of this is the situation in which some rhizobia use their own T3SS to enable their infection. Liu and colleagues identify a cellular target of the Mesorhizobium amphorae NopP effector, providing an important insight into the way in which endosymbionts use T3SS effectors to modulate the host response to allow infection.

Evolution of both the plant and microbe shapes the diverse landscape of interactions that we see today. The Pseudomonas flagellin protein flg22 acts as a potent microbe-associated molecular pattern, eliciting defense responses in plants-but not the flg22 from rhizobia. In this issue, Cheng and colleagues explore the evolution of flg22 immunogenicity, finding that flg22 from $\gamma$ - and $\beta$-Proteobacteria triggers a plant immune response, whereas flg22 from other proteobacterial classes do not. This observation raises an interesting question: why have plant pathogens with immunogenic flg22 not evolved nonimmunogenic versions? In a similar vein, Zhao and colleagues examine the conspicuous loss of the actin-like $m r e B$ gene from order Rhizobiales and suggest that this loss may have been a key step in the accommodation of rhizobia to an intracellular environment that includes antimicrobial host peptides.

Plants enter many diverse relationships with beneficial microbes that can improve plant health by increasing access to nutrients as well as by decreasing disease, either by promoting protective defense responses in the plant or via microbe-microbe interactions that directly counter potential pathogens. Pankievicz et al. review the extensive interactions of grasses with epiphytic and endophytic nitrogen-fixing bacteria that can contribute substantially to the nitrogen nutrition of the plant. Two other papers in this issue examine the complex metabolic linkages between endophytic fungi and their plant hosts. Frerigmann and colleagues examine the role of tryptophan metabolism, auxin signaling, and phosphate starvation responses in the interaction between Arabidopsis thaliana and the beneficial root-colonizing fungus Colletotrichum tofieldiae. Schweiger and colleagues report that interaction with beneficial, root-colonizing Trichoderma endophytes has profound effects on maize root metabolism, finding wide-reaching changes and revealing an important role for fungal iron regulation and terpene synthesis.

Soil microbial communities also interact with plant roots and can indirectly affect plant health. Feng and colleagues report the genome sequence of an Actinomycetes strain isolated from the rhizosphere that inhibits growth of the oomycete Phytophthora infestans, the causal agent of the devastating potato late blight. In addition, $\mathrm{Hu}$ and colleagues describe a soil microbial community that includes a rhizospheric fungus with seed mucilage degradation capabilities, associated with increased root nitrogen content, reflecting the importance of the plant-microbe-soil continuum.

We are pleased to present this focus issue addressing the top unanswered research question identified by the MPMI scientific community. We thank the authors for their valuable contributions addressing different aspects of this question and for making MPMI the forum for discussing these big ideas. We look forward to publishing more research on this topic over the coming years. Our 2022 special issue will focus on the question of next importance to the community, Top 10 question number 2: What is the Role of the Abiotic Environment on the Interactions between Plants and Microbes?

\author{
MPMI Editor-in-Chief Jeanne M. Harris \\ Guest Editors \\ Kathryn M. Jones, Dong Wang, and Alga Zuccaro
}

